\title{
Irrelevant reward and selection histories have different influences on task-relevant attentional selection
}

\author{
Mary H. MacLean • Barry Giesbrecht
}

Published online: 27 March 2015

(C) The Psychonomic Society, Inc. 2015

\begin{abstract}
Task-relevant and physically salient features influence visual selective attention. In the present study, we investigated the influence of task-irrelevant and physically nonsalient reward-associated features on visual selective attention. Two hypotheses were tested: One predicts that the effects of target-defining task-relevant and task-irrelevant features interact to modulate visual selection; the other predicts that visual selection is determined by the independent combination of relevant and irrelevant feature effects. These alternatives were tested using a visual search task that contained multiple targets, placing a high demand on the need for selectivity, and that was data-limited and required unspeeded responses, emphasizing early perceptual selection processes. One week prior to the visual search task, participants completed a training task in which they learned to associate particular colors with a specific reward value. In the search task, the reward-associated colors were presented surrounding targets and distractors, but were neither physically salient nor task-relevant. In two experiments, the irrelevant reward-associated features influenced performance, but only when they were presented in a task-relevant location. The costs induced by the irrelevant reward-associated features were greater when they oriented attention to a target than to a distractor. In a third experiment, we examined the effects of selection history in the absence of reward history and found that the interaction between task relevance and selection history differed, relative to when the features had previously been associated with reward. The results indicate that under conditions that demand highly efficient perceptual selection, physically nonsalient task-irrelevant and task-relevant factors interact to influence visual selective attention.
\end{abstract}

Keywords Selective attention · Attentional capture $\cdot$ Reward

M. H. MacLean $(\bowtie) \cdot$ B. Giesbrecht

Department of Psychological and Brain Sciences, University of

California Santa Barbara, Santa Barbara, California, USA

e-mail: mary.maclean@psych.ucsb.edu
Many accounts of selective attention have proposed that information-processing priorities are driven by the current task demands, goals, stimulus salience, or some combination thereof (e.g., Bundesen, 1990; Corbetta \& Shulman, 2002; Desimone \& Duncan, 1995; Itti \& Koch, 2001). However, recognition is growing that these explanations do not sufficiently account for the range of influences on selective attention (Anderson, 2013; Awh, Belopolsky, \& Theeuwes, 2012). For example, task switching (see Monsell, 2003) and intertrial priming (e.g., Belopolsky, Schreij, \& Theeuwes, 2010; Folk \& Remington, 2008; Sy, Elliott, \& Giesbrecht, 2013; Theeuwes, Kramer, \& Belopolsky, 2004) influence selective attention, suggesting that selection history is an important factor when setting priorities for selective information processing (Awh et al., 2012). Other evidence demonstrating that prior experience influences attention has included studies reporting that the visual selection of rewarded and reward-associated targets is more efficient than the selection of targets not associated with a reward (e.g., Della Libera \& Chelazzi, 2009; Kiss, Driver, \& Eimer, 2009; Raymond \& O’Brien, 2009), and studies reporting that performance is impaired when previously rewarded distractors are present in a display (e.g., Anderson et al., 2011; Anderson \& Yantis, 2012; Della Libera \& Chelazzi, 2009; Theeuwes \& Belopolsky, 2012). In other words, reward association, which is not always relevant to the current task, can influence the efficiency of selective attention. The purpose of the present work was to examine how task-irrelevant reward associations influence selective attention - specifically, whether the currently relevant task features modulate the effect of task-irrelevant reward associations.

\section{The role of currently relevant task demands}

Many aspects of the nature of visual selective attention have been debated (e.g., the units of selection, parallel vs. serial, etc.). One debate that is most relevant for the present work is 
the one regarding whether visual selective attention is driven predominantly by the bottom-up physical salience of object features (e.g., color, luminance; Theeuwes, 2010) or by topdown factors such as task relevance (e.g., Anderson \& Folk, 2012). There is evidence, however, that visual selection is driven by both of these factors, and furthermore by the interaction of salience and task relevance. In an experiment reported by Nordfang and colleagues (Nordfang, Dyrholm, \& Bundesen, 2013), participants were presented displays containing six letter targets and zero, two, or four digit distractors, and were asked to report as many letters as possible. The key manipulation was whether the target-defining task-relevant feature - that is, target (letter) or distractor (number) - was also physically salient (a color singleton). Using the theory of visual attention (TVA; Bundesen, 1990) computational framework to estimate the attentional weights for the different kinds of stimuli, Nordfang and colleagues observed that whole-report performance favored targets (letters $>$ numbers) as well as physically salient features (singleton $>$ nonsingleton). Furthermore, they discovered that the effect of the singleton was larger for the target than for the distractor. These results are consistent with the notion that visual selection is not driven by salience or task relevance alone, but rather that the interaction between these factors plays a key role in modulating the efficiency of selective attention.

\section{Irrelevant learned reward association}

In addition to the evidence that visual selection is driven by both task relevance and salience (Nordfang et al., 2013), there is evidence that visual selection is also driven by taskirrelevant factors that are not physically salient. One factor that has received some attention in the recent literature is the previously learned association of a reward with a currently task-irrelevant feature (see Anderson, 2013, for a review).

An irrelevant learned reward association is an association between a feature (e.g., color) and reward that is irrelevant to the current task. Importantly, both the feature and the reward that it is associated with are irrelevant to the current task, since the target-defining task-relevant feature is orthogonal to the reward-associated feature (e.g., shape; Anderson et al., 2011). These irrelevant reward-associated features can interfere with currently task-relevant-driven visual selection, resulting in distraction (e.g., Anderson et al., 2011; Anderson \& Yantis, 2012; Della Libera \& Chelazzi, 2009; Theeuwes \& Belopolsky, 2012). Specifically, when the task-irrelevant reward-associated feature is present, the ability to attend to orthogonal task-relevant features is typically impaired, as indicated by increased reaction times (RTs) to identify the target, as compared to when the irrelevant reward-associated feature is absent (Anderson et al., 2011).

The effects of irrelevant reward associations on performance are partly due to the influence on spatial selectivity, and this influence is thought to represent an effect of the irrelevant reward association on the orienting of spatial attention. For instance, people are slower to attend to locations where the irrelevant learned reward-associated feature appeared previously (inhibition of return; Anderson et al., 2011), and people are more likely to make overt shifts of attention (eye movements) to locations with irrelevant learned reward-associated features than to locations with other task-irrelevant features (Anderson \& Yantis, 2012; Theeuwes \& Belopolsky, 2012). Similarly, physically salient features also affect the orienting of spatial attention and can interfere with the selection of taskrelevant features (e.g., Theeuwes, 1992). However, irrelevant reward associations can capture attention even in the presence of a physically salient feature (e.g., shape singleton; Anderson et al., 2011).

Together, this evidence suggests that task-irrelevant factors modulate visual selection, particularly spatial selection, and that these factors compete with those of physical salience and task relevance. However, two aspects concerning the influence of task relevance on the effect of task-irrelevant reward associations remain unclear. First, an effect of irrelevant reward associations on attention has been shown when the reward-associated feature has oriented attention to either a distractor (e.g., Anderson et al., 2011) or a target (e.g., Della Libera \& Chelazzi, 2009). However, these experiments did not examine whether irrelevant reward associations affect visual selection independently of the current task relevance or whether irrelevant reward associations interact with current task relevance to determine the efficiency of visual selection.

Second, recent investigations have shown that the reward history at particular spatial locations can influence the effect of irrelevant reward associations on attention (e.g., Chelazzi et al., 2014; Hickey, Chelazzi, \& Theeuwes, 2014). However, the influence of the task relevance of the spatial location at which the reward-associated feature is presented has yet to be examined. Thus, it is unclear to what extent the task relevance of specific spatial locations affects the influence of the reward association. This is a particularly important issue to address because the effect of irrelevant learned reward appears to be, at least in part, spatial (Anderson et al., 2011; Anderson \& Yantis, 2012; Theeuwes \& Belopolsky, 2012).

\section{Present study}

In the present study, we addressed the issue of the relationship between the target-defining task-relevant and task-irrelevant reward-associated features on visual selection, using a design that demanded a high level of selectivity and emphasized the role of early selection processes. In order to determine the relationship between the influences of the task-relevant and irrelevant reward-associated features on visual selection, these factors were manipulated independently in the present study. 
The combination of these factors meant that an object's taskrelevant features could define it as a target or a distractor in either a task-relevant or a task-irrelevant location, and an object's task-irrelevant feature could have previously been associated or not associated with a reward. This design allowed us to determine whether these factors influence performance independently or dependently.

Within the context of this design, we examined two alternative hypotheses about the manner in which the currently target-defining task-relevant and -irrelevant reward-associated features combine to influence visual selection. One hypothesis is that the effects of these features on attention are dependent on each other (i.e., they interact), and the other is that the effects of these features are independent. Both hypotheses predict benefits to behavioral performance when the irrelevant reward-associated feature orients attention to a to-be-reported item (i.e., a probed target) in a partial-report task, and costs when the reward-associated feature orients attention elsewhere (including to nonprobed targets, nontarget distractors, or flankers). These hypotheses differ according to the pattern of performance costs. If the influence of irrelevant reward association is dependent on the task-relevant features, then the relative sizes of the costs should differ according to those target-defining features: The costly effect of irrelevant reward associations should be larger for nonprobed targets than for distractors. Furthermore, the costs should be smallest for stimuli presented in task-irrelevant locations - that is, where a tobe-reported target never appears (flankers). If the effect of reward history on attention is independent of task relevance, then an irrelevant learned reward would affect early visual selection equivalently, regardless of the task-relevant feature.

\section{Experiment 1}

In Experiment 1, we examined the effect of irrelevant reward association as a function of task relevance in an attention task involving visual search for target letters (three) amongst distractor digits in the face of task-irrelevant stimuli (flankers). Irrelevant reward associations between a specific color (red or blue) and reward value (\$0.05 or $\$ 0.01)$ were learned in a training task one week prior to completing the attention task. Importantly, the features that were associated with reward during the training task were both irrelevant and physically nonsalient in the attention task.

\section{Method}

\section{Participants}

Twenty-nine undergraduate students volunteered to participate in this study $\left(M_{\text {age }}=20 ; 18\right.$ females, 11 males $)$. Twelve were independent-studies students, and 17 were recruited from the Psychological and Brain Sciences research participation pool at the University of California, Santa Barbara. All received research participation credit. All procedures conformed to a protocol approved by the University of California, Santa Barbara, Human Subjects Committee.

Learned reward association training task

Stimuli The stimuli consisted of six differently colored rings $\left(0.78^{\circ}\right.$ diameter $)$ presented on the arc of an imaginary circle (radius $=1.31^{\circ}$ from the center of the screen to the center of each circle) and equidistant from each other $\left(1.45^{\circ}\right.$ center-tocenter). The colors of the rings were red (RGB: 206, 0, 0), blue $(0,0,250)$, orange $(197,98,0)$, teal $(0,118,118)$, brown $(169$, $85,0)$, green $(0,141,0)$, gold $(118,118,0)$, violet $(128,0$, $192)$, and gray $(110,110,110)$. A white $(255,255,255)$ line segment $\left(0.47^{\circ}\right.$ long, $0.05^{\circ}$ thick $)$ was presented centered inside each colored ring, and the orientation of each segment was oblique $\left(45^{\circ}\right.$, left or right), horizontal, or vertical.

Procedure Each trial of the training task began with a white fixation cross $\left(0.26^{\circ}\right.$ of visual angle width/height $)$ presented in the center of the display. The duration of the fixation cross, determined randomly on each trial, was either 400,500 , or $600 \mathrm{~ms}$. The stimulus display followed the fixation cross and was presented for $600 \mathrm{~ms}$. The stimulus display (Fig. 1a) consisted of one target ring and five distractor rings. The target ring was red on half of the trials and blue on the other half of the trials. The color of each distractor ring was drawn randomly without replacement on each trial from the remaining colors (orange, teal, brown, green, gold, violet, and gray). The location of the target ring was determined randomly without replacement from the six possible locations on each trial.

The line segment within the target ring was horizontal on half of the trials and vertical on the other half of the trials. The line segments within the distractor rings were tilted $45^{\circ}$ to either the left or the right. Participants were instructed to press " $z$ " on a keyboard if the line segment within the target ring was vertical, and " $\mathrm{m}$ " if it was horizontal. The participants were instructed to make this response "as quickly as possible while still being accurate." Once a response was made, a feedback display was then presented for 1,500 ms. The feedback display showed the amount of money won on that trial in the center of the screen, with the total accumulated presented below that. Participants could only receive a reward on correct trials, although they were not informed of this fact. On highreward trials, participants could win $\$ 0.05$, and $80 \%$ of the high-reward trials had the potential to be rewarded (i.e., if performance was correct). On low-reward trials, participants could win $\$ 0.01$, and $20 \%$ of the low-reward trials had the 
(a)

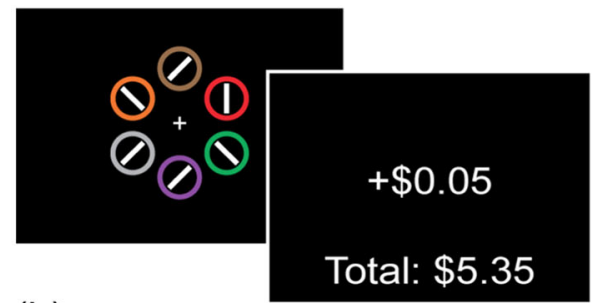

(b)

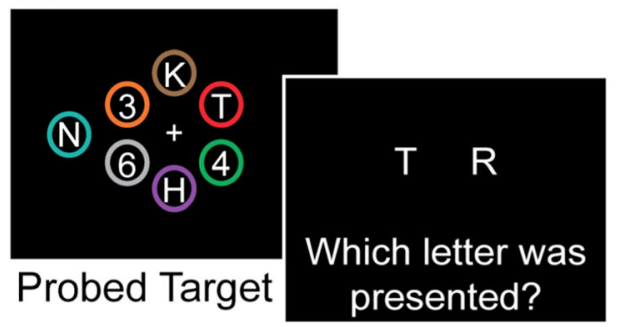

Fig. 1 Trial examples from Experiment 1. (a) The irrelevant-reward training task, with a feedback display for a correct trial. (b) The attention task, with a probed-target trial presented. In the probed-target condition, the letter $\mathrm{T}$, a subsequently probed target, is presented inside either a red or a blue, and thus a reward-associated, circle. All other items are presented inside differently colored circles that are neither red nor blue. (c) Examples of the other experimental conditions. In the other-

potential to be rewarded. The target color (i.e., red or blue) associated with the high reward was counterbalanced across participants. Participants were instructed that they had the chance to win money on each trial and that they would be paid the total amount they had accrued over the course of the experiment. On average, participants won and were paid $\$ 11.00$.

The training task began with 20 practice trials that were not rewarded, followed by ten blocks of 80 trials, for a total of 800 trials, consisting of 400 high-reward and 400 low-reward trials. High- and low-reward trials were mixed randomly within each block of the training task and were distributed evenly within each block.

The percentages of correct line segment orientation discriminations (i.e., horizontal vs. vertical) were measured for high- and low-reward trials. RTs on correct trials were measured relative to the onset of the stimulus display for high- and low-reward trials separately.

\section{Attention task}

Stimuli The six colored rings used in the training task were also used in the attention task. An additional flanker ring was presented to the left of the task array on half of the trials, and to the right on the other half of the trials. The flanker appeared $3.12^{\circ}$ center-tocenter from the fixation cross and $1.95^{\circ}$ center-to-center from the nearest task array ring. In the six circles presented equidistant from fixation, three contained letters $\left(0.26^{\circ}\right.$ width, $0.31^{\circ}$ height $)$ drawn randomly on each trial from among the following: A, E, F, H, J, K, $\mathrm{L}, \mathrm{M}, \mathrm{N}, \mathrm{P}, \mathrm{R}, \mathrm{T}, \mathrm{U} \mathrm{V}, \mathrm{X}$, and Y. Other letters were excluded due to (c)

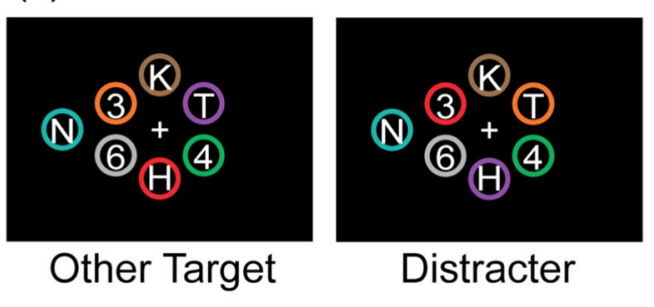

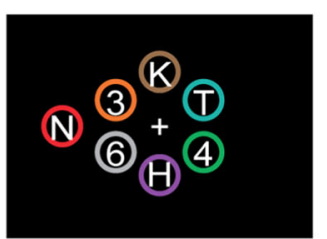

Flanker

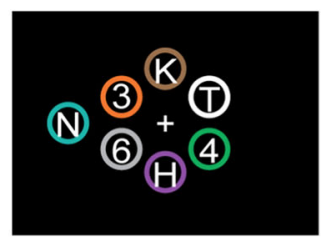

Neutral Baseline target condition example, the letter $\mathrm{H}$, a target that is not subsequently probed, is presented inside the reward-associated circle. In the distractor condition example, the number 3, a distractor, is presented inside the reward-associated circle. In the flanker condition example, the flanking item, the letter N, is presented inside the reward- associated circle. Finally, in the neutral-condition example, no reward-associated (i.e., red or blue) circle is present

their similarities with certain numbers. The remaining three circles contained numbers $\left(0.26^{\circ}\right.$ width, $0.31^{\circ}$ height $)$, drawn randomly on each trial from among the numbers 1-9. Masks were the symbols \#, \%, @, and \&, which were presented in white $(255,255$, 255) font on top of each other to create a pattern mask replacing the letters and numbers in each of the rings. The colored rings were also replaced with white rings during the mask.

Procedure Each trial of the attention task began with a white fixation cross presented in the center of the display. The fixation was displayed until the participant pressed the spacebar to show the stimulus display. The participants were instructed to wait until they were fixated on the fixation cross before initiating the stimulus display, and to maintain fixation on the fixation cross whenever it was displayed, including during the stimulus display. The stimulus display followed the fixation cross and was presented for $232 \mathrm{~ms}$; each stimulus was then masked for $250 \mathrm{~ms}$, and then the response display was presented until response. The short display duration and mask were both aspects of this task's data-limited design. The response display consisted of two letters: the probed target and a lure letter. The probed target was one of the letters presented in the task array, and the lure was a letter not presented in the stimulus display. Participants were instructed to indicate which of the letters was one of the letters presented in the task array by pressing that letter's key on the keyboard (target identification task). They were instructed to try to be as accurate as possible and to make their "best guess" if they were unsure. Each trial was separated by a 500-ms intertrial interval, 
during which the fixation cross remained on the screen. An example of the stimulus display is shown in Fig. $1 b$.

Participants were instructed to pay attention to the letters (targets) and to ignore the numbers (distractors). Importantly, they were also told that the color of the rings was irrelevant to the task and to be ignored, as was the flanker. For a subset of participants $(n=12)$, the flanker ring always contained a letter, and thus was always congruent with the target; for the rest of the participants, the flanker was a letter, a number (distractorcongruent), or a symbol (neutral) with equal probabilities. ${ }^{1}$

The attention task began with ten practice trials, followed by ten blocks of 100 trials, for a total of 1,000 trials: 800 reward-associated and 200 neutral trials. The rewardassociated trials were trials on which one of the rings was blue or red. Half of the reward-associated trials were high-reward association trials, on which the high-reward color for that participant (determined in the training task) was present, and the other half were low-reward association trials. Neutral trials were trials on which neither reward-associated color was present- that is, no blue or red ring. Note that no rewards were given in the attention task. The reward association refers to the learned association from the training task, but it was irrelevant to the attention task.

Design The attention task had two within-subjects factors: previous reward size and task relevance. There were two sizes of previous rewards in the attention task - high or low. Also, four levels of task relevance in the attention task were determined by which category of task-relevant feature was presented inside the reward-associated colored ring (see Figs. $1 \mathrm{~b}$ and c). On probed-target and other-target trials, a target (a letter) was presented in a task-relevant location (in the task array) inside the reward-associated (red or blue) ring. These trials had identical task arrays, but differed in terms of the response alternatives shown, such that the probed target was the target probed for response, whereas other targets were not. On the distractor trials, one of the distractors (a number) was presented in a task-relevant location (in the task array) inside the reward-associated ring. On the flanker trials, a letter, or for a subset of participants a number or symbol, was presented in a task-irrelevant location (outside the task array), inside the

\footnotetext{
${ }^{1}$ The identity of the flanker item was varied for some participants in order to examine whether the identity of the flanker influenced whether the irrelevant reward association would orient attention to the flanker item; for example, perhaps the irrelevant reward association might only orient attention outside of task-relevant space when the flanker item was targetcongruent (i.e., a letter). However, we observed no differences in the patterns of any of the effects reported in Experiment 1 between the participants for whom flanker identity varied and those for whom it was always target-congruent. Furthermore, within those participants for whom the flanker identity varied, there were no significant effects of flanker identity. Thus, flanker identity did not interact with the effects of irrelevant reward-associated features on attention.
}

reward-associated ring. The factorial combination of relevance and reward association size resulted in eight experimental conditions. In each experimental condition, 100 trials were presented, with ten trials/block, intermixed randomly within each block.

\section{Procedure}

Participants completed the learned reward association training and the attention task in two separate sessions, exactly one week apart. Before completing the experiment, participants were informed that the experiment concerned attention; they were not informed prior to training that they could win money as part of the experiment.

\section{Apparatus}

Both the training and attention tasks were run using custom scripts with functions from the Psychtoolbox (PTB-3), using MATLAB R2013a installed on a Mac Mini running OS X and were presented on CRT monitors $(36 \times 27 \mathrm{~cm})$ with a resolution of $1,280 \times 1,024$ and a refresh rate of $60 \mathrm{~Hz}$. All stimuli were viewed from a distance of $110 \mathrm{~cm}$ and were presented on a black (RGB: 0, 0,0) background.

\section{Results}

Learned reward association training task

Reaction time A paired $t$ test was used to examine RTs on correct trials as a function of reward size (high or low). RTs were significantly faster on high-reward $(M=435 \mathrm{~ms}, S E M=$ $6 \mathrm{~ms})$ than on low-reward $(M=449 \mathrm{~ms}, S E M=6 \mathrm{~ms})$ trials, $t(28)=5.76, p<.001$; see Fig. 2 a.

Accuracy A similar paired $t$ test was used to analyze the accuracy data. Accuracy did not differ significantly between high-reward $(M=75 \%, S E M=2 \%)$ and low-reward $(M=$ $74 \%, S E M=2 \%)$ trials, $t(28)=1.47, p=.153$; see Fig. 2 b.

\section{Attention task}

Hypotheses Both hypotheses outlined in the introduction predicted that reward associations should affect target performance. Specifically, the irrelevant reward-associated feature should result in a benefit to performance in the probed-target conditionthat is, higher accuracy in the probed-target condition than in the neutral baseline condition. Furthermore, the irrelevant rewardassociated feature should result in a cost to performance in the other-target, distractor, and flanker conditions - that is, lower accuracy in these conditions than in the neutral baseline condition. In order to provide evidence that reward history and not selection history was driving performance (since both reward- 
(a)

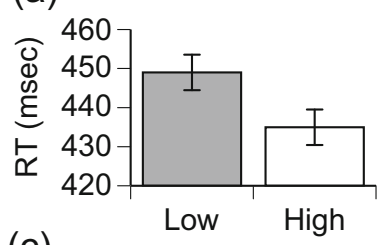

(c)

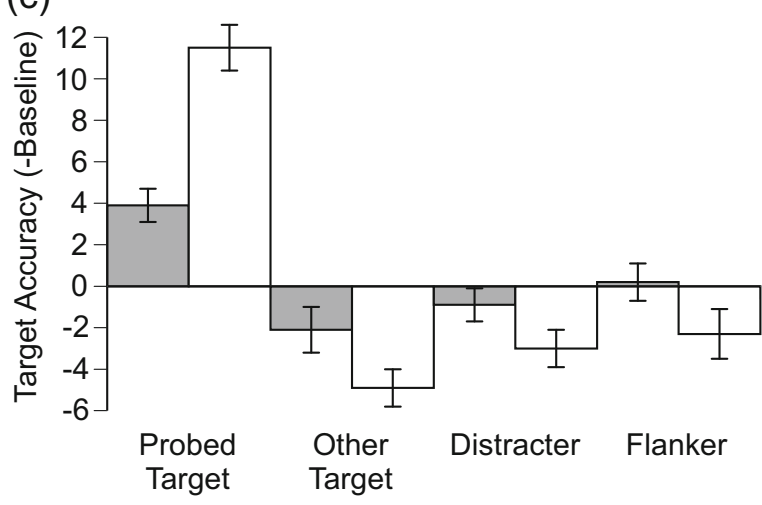

Low Reward Association

High Reward Association

Fig. 2 Results of Experiment 1: (a) reaction time (RT) results from the training task, (b) accuracy results from the training task, and (c) baseline subtracted target accuracy results according to task relevance (probed target, other target, distractor, or flanker) and the size of the reward association (low or high). Error bars represent standard errors of the mean differences ( $\mathbf{a}$ and $\mathbf{b})$ and standard errors of the means (c)

associated features were also previously target features), it was necessary to demonstrate that the effects of the reward-associated feature were different for those features associated with a high magnitude of reward than for those associated with a lower magnitude of reward. The typical finding is that the effect of the highmagnitude reward-associated feature is reliable (i.e., significantly different from neutral), whereas the effect of the low-magnitude reward-associated feature is not (e.g., Anderson et al., 2011).

The hypotheses differed in terms of the predicted patterns of the observed costs. According to the dependent hypothesis, costs should change with the category of the task-relevant feature; the largest costs should be observed in the othertarget conditions, and the smallest costs in the flanker condition. In contrast, according to the independent hypothesis, the costs should not differ with task relevance. Again, in order to conclude that the effect of the irrelevant reward history was what modulated performance, and not the irrelevant selection history, we would need to show that the difference in the effects of high- and low-reward-associated features differed according to task relevance. If this were not the case, it would suggest that irrelevant selection history and not reward history varied as a function of task relevance.

Effect of irrelevant reward association: Costs and benefits Accuracy was significantly above chance in all experimental conditions and on neutral baseline trials (all $p \mathrm{~s}<.001)$. In order to determine whether there was a significant effect of irrelevant reward associations, the target accuracy on trials in which a reward-associated color was present (probe target, other target, distractor, flanker) was compared to the target accuracy on neutral baseline trials, in which no reward-associated color was present, using paired $t$ tests (see Table 1).

For both low and high irrelevant reward associations, we observed a benefit to target accuracy on the probed-target trials and a cost to target accuracy on the other-target, distractor, and flanker trials, as compared to neutral trials (although the result was not significant in all conditions). The effect of irrelevant reward was significant for all irrelevant high-rewardassociated conditions except the flanker condition. The irrelevant low-reward association only had a significant effect in the probed-target condition. Consistent with our hypothesis, and similar to previous studies (i.e., Anderson et al., 2011), reliable effects emerged for irrelevant high- but not lowreward-associated features. However, neither high- nor lowreward-associated features had a reliable effect on performance in the flanker condition. It is possible that the lack of effects in the flanker condition was due to its increased distance from fixation relative to the other stimuli, although effects of flankers have certainly been documented at distances from fixation even greater than those used here (e.g., $>5 \mathrm{deg}$ of visual angle; Kyllingsbæk, Sy, \& Giesbrecht 2011).

Influence of task relevance on the costs of irrelevant reward association Although the above results demonstrate the expected effect of irrelevant reward association on performance, in order to test the dependent versus independent hypotheses, we investigated whether this effect was influenced by task relevance. Specifically, whether the costs associated with the irrelevant reward association changed as the task-relevant feature changed (i.e., other target $>$ distractor $>$ flanker). In order to investigate this possibility, the cost of the irrelevant reward association was computed as a difference measure by subtracting accuracy on the neutral baseline trials from accuracy on the other-target, distractor and flanker trials (see Fig. 2c). These data were analyzed in a repeated measures analysis of variance (ANOVA) as a function of the current task relevance (other target, distractor, or flanker) and the size of the irrelevant reward association (high or low). Both models predicted costs in performance relative to baseline; however, using the difference scores, only the dependent model predicted a main effect of task relevance.

The main effect of task relevance was significant $\left[F(2,56)=4.39, p=.017, \eta^{2}=.14\right]$, such that the cost of the irrelevant reward association differed according to task relevance. As hypothesized, the size of the costs changed numerically as the task-relevant feature changed, such that the costs were largest for the othertarget condition, smaller for the distractor condition, and 
Table 1 Mean target accuracy with standard error and t-value (paired comparison with target accuracy in neutral condition) for each experimental condition, within each Experiment

\begin{tabular}{|c|c|c|c|c|c|c|c|c|c|}
\hline & \multicolumn{3}{|c|}{ Experiment 1} & \multicolumn{3}{|c|}{ Experiment 2} & \multicolumn{3}{|c|}{ Experiment 3} \\
\hline & $M$ & SEM & $t(28)$ & $M$ & SEM & $t(17)$ & $M$ & SEM & $t(16)$ \\
\hline High/red probed & 82.82 & 1.26 & $10.21^{* * * *}$ & 71.61 & 2.56 & $2.68^{*}$ & 72.44 & 2.29 & $3.19^{* *}$ \\
\hline Low/blue probed & 74.98 & 1.61 & $4.59^{* * *}$ & 69.86 & 1.71 & $2.89^{*}$ & 69.55 & 2.01 & $2.21^{*}$ \\
\hline High/red other & 66.28 & 1.77 & $5.28^{* * *}$ & 63.33 & 1.78 & 1.67 & 65.74 & 1.36 & 0.76 \\
\hline Low/blue other & 69.20 & 1.65 & 1.81 & 64.05 & 1.99 & 1.28 & 63.83 & 1.71 & $2.69^{*}$ \\
\hline High/red distractor & 68.07 & 1.51 & $3.44^{* *}$ & 61.39 & 1.61 & $3.88^{* *}$ & 62.58 & 1.68 & $2.99^{* *}$ \\
\hline Low/blue distractor & 70.43 & 1.73 & 0.87 & 66.78 & 2.11 & 0.81 & 64.65 & 2.19 & 1.42 \\
\hline High/red flanker & 68.88 & 1.56 & 1.95 & 66.12 & 1.77 & 0.24 & 67.97 & 1.81 & 0.89 \\
\hline Low/blue flanker & 71.48 & 1.62 & 0.35 & 66.58 & 2.06 & 0.61 & 67.37 & 1.63 & 0.52 \\
\hline Neutral & 71.17 & 1.42 & & 65.74 & 1.71 & & 66.69 & 0.52 & \\
\hline
\end{tabular}

smallest for the flanker conditions. Post-hoc pair-wise comparisons of the factors contributing to the main effect of task relevance indicated that target performance costs in the other-target and flanker conditions were significantly different from each other, such that costs in the other-target condition were significantly larger than those in the flanker condition $\left(M_{\text {diff }}=2.4 \%, p\right.$ $=.002)$. However, the costs in the distractor condition were not significantly different from those in either the other-target $\left(M_{\text {diff }}=\right.$ $1.5 \%, p=.126)$ or the flanker $\left(M_{\text {diff }}=0.9 \%, p=.258\right)$ condition.

The main effect of size of the irrelevant reward association was significant $\left[F(1,26)=10.30, p=.003, \eta^{2}=.27\right]$, such that the costs were larger for irrelevant high-reward associations than for irrelevant low-reward ones. The interaction between task relevance and the size of the irrelevant reward association was not significant $\left[F(2,56)=0.07, p=.937, \eta^{2}<.01\right]$, indicating that although costs were larger for irrelevant high-reward associations than for irrelevant low-reward associations, this difference was not modulated by task relevance (see Fig. 2c). This suggests that although there is a decrease in the size of the costs as task relevance decreases (other target $>$ distractor $>$ flanker), this effect may reflect the moderating influence of task relevance on the effect of irrelevant selection history, and not of irrelevant reward history alone.

\section{Experiment 2}

In Experiment 1, the effect of irrelevant reward associations was examined as a function of task relevance and the size of the irrelevant reward association. The irrelevant reward association resulted in a benefit to performance when it was associated with the probed target, and a cost to performance when it was associated with any other object except the flanker item. Moreover, the costly effect of irrelevant reward association differed according to task relevance. The pattern of results indicated dependent effects of the current task relevance and irrelevant reward associations, such that the performance costs caused by irrelevant reward association were numerically largest for targets, smaller for distractors, and smallest for objects presented outside of task-relevant space (flankers). However, because these effects were equivalent for both high- and low-reward-associated features, it was possible that irrelevant selection history, and not only reward history, was modulated by task relevance. These results do, however, suggest that irrelevant reward associations did not have a reliable effect on visual selection when presented outside of task-relevant space.

The purpose of Experiment 2 was to further investigate the factor of task-relevant versus -irrelevant space. Specifically, in Experiment 1 we found no significant effect of irrelevant reward associations on flanker trials. However, not only did the flanker differ from other objects because of its spatial location (i.e., it was always in a task-irrelevant location), it also differed from other objects in that it appeared in a location that had never previously been associated with reward, because there no flanker object had appeared in the training task. All other objects (probed targets, other targets, and distractors) appeared in locations that had previously been reward-associated. Thus, it is possible that the lack of an effect of the irrelevant reward association in the flanker conditions in Experiment 1 was due to the fact that the flanker had never appeared in a previously reward-associated location. It is also possible that appearing in a previously reward-associated location contributed to the significant effect of the irrelevant reward association for the other objects. To address this issue, the locations of the rings in the training task and the attention task were arranged so that all objects, including the flanker, could appear in a location (relative to fixation) that previously either had or had not been rewarded in the training task.

\section{Method}

Except where indicated, Experiment 2 was identical to Experiment 1. 


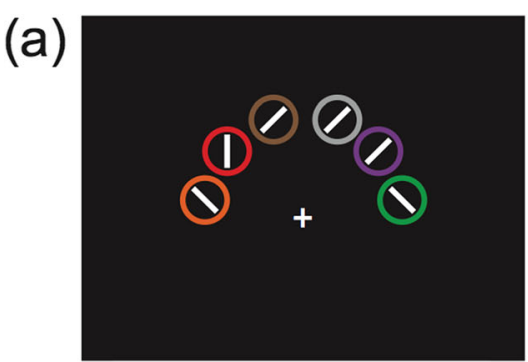

Fig. 3 Trial examples from Experiment 2: (a) the irrelevant-reward training task and (b) the attention task. The locations within the white dashed boxes are locations that had not previously been rewarded in the

\section{Participants}

Sixteen undergraduate students volunteered to participate in this study $\left(M_{\text {age }}=19\right.$; ten females, six males). ${ }^{2}$ Two were independent-study students, and 14 were recruited from the Psychological and Brain Sciences research participation pool at the University of California, Santa Barbara. All procedures conformed to a protocol approved by the University of California, Santa Barbara, Human Subjects Committee.

\section{Learned reward association training task}

The training task was identical to that in Experiment 1, except for the location of the colored rings. In Experiment 2, the six colored rings appeared in an arc presented either above or below the fixation cross (see Fig. 3a). The position of the arc was counterbalanced across participants. The colored rings $\left(0.63^{\circ}\right.$ diameter) were presented equidistant from the fixation cross $\left(1.86^{\circ}\right.$ center-to-center $)$ and from each other $\left(0.95^{\circ}\right.$ center-to-center). The line segments were identical to those presented in Experiment 1, except that they were slightly shorter $\left(0.38^{\circ}\right)$ in order to fit inside the slightly smaller rings.

\section{Attention task}

The six colored rings of the task array were presented in an arc to the left of fixation for half of the blocks of trials, and to right of the fixation cross for the other half of the blocks of trials (the size and relative locations of the task array were identical to those aspects in the training task). The flanker was presented in the opposite half of the visual field and either above or below fixation $\left(1.86^{\circ}\right.$ center-to-center, relative to the fixation cross), in a location identical to one of those used in the training task $\left(1.84^{\circ}\right.$ center-to-center, relative to the closest task array object). Thus, when an object appeared in the same half

\footnotetext{
${ }^{2}$ The difference in sample sizes between Experiments 1 and 2 is due to collapsing across the subsamples $(n=12$ and 17) in Experiment 1 , in that the two subsamples differed in terms of whether the flanker was always congruent or could be congruent, incongruent, or neutral, relative to the target category (see note 1). We found no difference in the patterns of results between the two subsamples of Experiment 1.
}

(b)

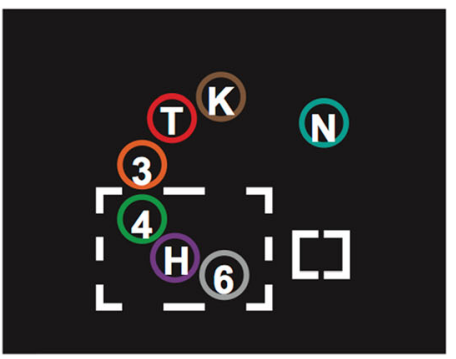

irrelevant-reward training task. In this example, the flanker item appears in a location that had previously been rewarded in the irrelevant-reward training task

of the visual field (i.e., top or bottom) as the arc in the training task, it was in a reward-associated location. When an object appeared in the opposite half of the visual field from the arc in the training task, it was not in a reward-associated location. A sample attention task display is shown in Fig. 3b. The attention task consisted of ten practice trials and nine blocks of 120 trials, for a total of 1,080 trials: 864 reward-associated and 216 neutral trials.

\section{Results}

Learned reward association training task

Reaction time A paired $t$ test was used to examine the RTs on correct trials as a function of reward size (high or low). RTs were not significantly faster on high-reward $(M=427 \mathrm{~ms}$, $S E M=14 \mathrm{~ms})$ than on low-reward $(M=436 \mathrm{~ms}, S E M=$ $15 \mathrm{~ms}$ ) trials, $t(15)=2.01, p=.062$; see Fig. $4 \mathrm{a}$.

Accuracy A similar paired $t$ test was used to analyze the accuracy data. Accuracy was significantly better on high-reward $(M=66 \%, S E M=3 \%)$ than on low-reward $(M=60 \%$, $S E M=3 \%)$ trials, $t(15)=3.13, p=.007$; see Fig. $4 \mathrm{~b}$.

\section{Attention task}

Effect of irrelevant reward association: Costs and benefits Accuracy was significantly above chance in all experimental conditions and on neutral baseline trials (all $p \mathrm{~s}<$ .001). As in Experiment 1, there was a benefit to target accuracy on the probed-target trials and a cost to target accuracy on the other-target trials, as compared to neutral trials. Only the high-reward-associated distractor condition demonstrated a cost, whereas the low-reward distractor and both flanker conditions did not. The effect of irrelevant reward was significant for all irrelevant high-reward-associated conditions except for the other-target and flanker conditions. None of the irrelevant low-reward associations had a significant effect (see Table 1).

Influence of task relevance on costs of irrelevant reward association The mean baseline subtracted accuracy, displayed 
(a)

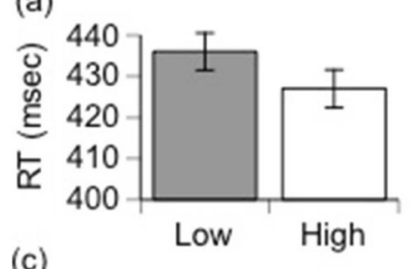

(b)

(c)

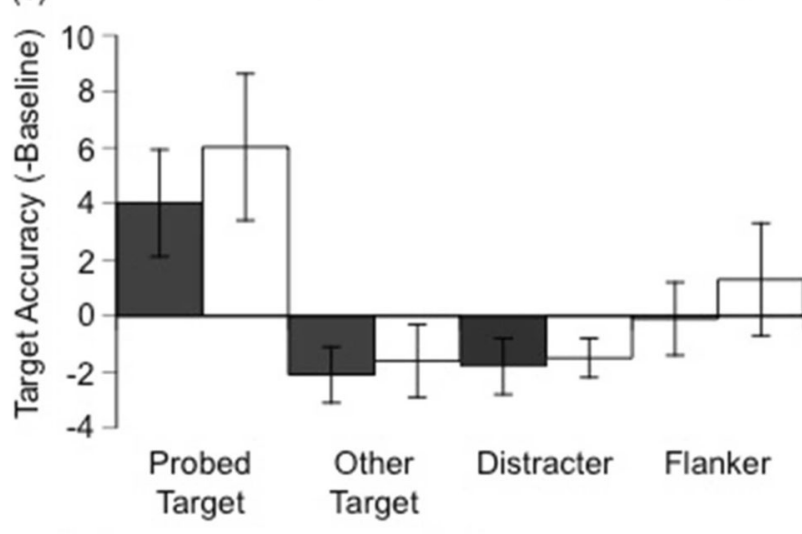

Rewarded Location

Non-rewarded Location

Fig. 4 Results of Experiment 2: (a) reaction time (RT) results from the training task, (b) accuracy results from the training task, and (c) baseline subtracted target accuracy results according to task relevance (probed target, other target, distractor, or flanker) and the reward history of the location (rewarded or nonrewarded location). Error bars represent standard errors of the mean differences ( $\mathbf{a}$ and $\mathbf{b}$ ) and standard errors of the means (c)

for task relevance by reward-history-of-location conditions, is shown in Fig. 4c. As in Experiment 1 the main effect of task relevance was significant $\left[F(2,30)=3.39, p=.047, \eta^{2}=.18\right]$. Also as in Experiment 1, the costs were numerically largest for the other-target condition, smaller for the distractor condition, and smallest for the flanker conditions (Fig. 4c). As in Experiment 1, post-hoc pair-wise comparisons of the factors contributing to the main effect of task relevance indicated that target performance costs in the other-target and flanker conditions were significantly different from each other, such that costs in the other-target condition were significantly larger than those in the flanker condition $\left(M_{\mathrm{diff}}=2.5 \%, p=.017\right)$. However, the costs in the distractor condition were not significantly different from those in either the other-target $\left(M_{\text {diff }}=\right.$ $0.2 \%, p=.831)$ or the flanker $\left(M_{\text {diff }}=2.3 \%, p=.075\right)$ condition. No other main effects or interactions were significant, including those of reward magnitude (high vs. low; all $p \mathrm{~s}>.08$ ).

Effects of reward-associated location In the above analysis, we found no main effect of the reward history of the location on the effect of the irrelevant reward-associated feature, nor any interaction with the effect of task relevance (see Fig. 4c). Thus, we did not find any evidence that the effects of irrelevant reward association on early visual selection, or the interaction of task relevance and irrelevant reward association on early visual selection, were dependent on the reward history of task-relevant spatial locations. Furthermore, we continued to find no effect of irrelevant reward history for objects appearing outside of task-relevant space (i.e., flankers). There was no significant effect of the irrelevant reward association in any of the flanker conditions (i.e., relative to the neutral baseline condition), whether or not the flanker was presented in a previously rewarded location (all $p \mathrm{~s}>.542$ ).

\section{Experiment 3}

In both Experiments 1 and 2, we observed that irrelevant reward history influenced performance reliably when high- but not low-reward-associated features were presented within task-relevant space, but not in a task-irrelevant flanker location. We also observed that the costs to performance of the reward-associated features decreased with task relevance. However, because such decreases were equivalent across high- and low-reward-associated features, it was unclear to what extent the interaction with task relevance was being driven by irrelevant reward history, irrelevant selection history (i.e., that both features had formerly been target features during training), or both. To further investigate this possibility, we performed a control experiment. This experiment was identical to Experiment 1, except that there were no rewards and no reward contingencies in the training task. Thus, any effects of the presence of those features in the subsequent attention task would be attributable to irrelevant selection history, not to reward history. More importantly, because the effects in Experiment 1 could be attributed to a combination of reward and selection history, whereas the effects observed in Experiment 3 could be attributed to selection history alone, any differences between Experiments 1 and 3 would likely be due to reward history.

\section{Method}

Participants

Seventeen undergraduate students volunteered to participate in this study $\left(M_{\text {age }}=19\right.$ years; six females, 11 males). All were recruited from the Psychological and Brain Sciences research participation pool at the University of California, Santa Barbara. All procedures conformed to a protocol approved by the University of California, Santa Barbara, Human Subjects Committee. 


\section{Results}

Learned reward association training task

Reaction time A paired $t$ test was used to examine the RTs on correct trials as a function of target color (red or blue). RTs were significantly faster on blue-target $(M=481 \mathrm{~ms}, S E M=$ $6 \mathrm{~ms})$ than on red-target $(M=485 \mathrm{~ms}, S E M=6 \mathrm{~ms})$ trials, $t(16)=2.91, p=.01$. This small $\left(M_{\text {diff }}=4 \mathrm{~ms}, S E M_{\text {diff }}=1 \mathrm{~ms}\right)$ but reliable difference suggests that blue targets were perhaps more salient than red targets.

Accuracy A similar paired $t$ test was used to analyze the accuracy data. Accuracy was not significantly different on redtarget $(M=71 \%, S E M=2.32 \%)$ and blue-target $(M=71 \%$, $S E M=2.37 \%$ ) trials, $t(16)=-0.00, p=.909$.

\section{Attention task}

Effect of irrelevant selection history: Costs and benefits Accuracy was significantly above chance in all experimental conditions (probed target, other target, distractor, or flanker $\times$ red vs. blue former target feature) and on neutral baseline trials (all $p s<.001$; see Table 1 ). We found a benefit to target accuracy on the probed-target trials and a cost to target accuracy in all other conditions, as compared to neutral trials (although the result was not significant in all conditions). In the probed-target condition, the presence of both the red and blue former target, but currently irrelevant, features significantly improved performance relative to performance in the neutral condition. In the other-target condition, only the blue former target feature had a significant effect, whereas in the distractor condition, only the red former target feature had a significant effect. As in the results of Experiment 1, there were no significant effects of either former target feature on performance in the flanker condition. Thus, unlike the findings of Anderson et al. (2011) in a similar control experiment, our task-irrelevant and physically nonsalient former target features did capture attention one week later in a different task. Although the training task in our experiment was very similar to that used by Anderson et al., our attention task differed in several ways. It is possible that the effects of irrelevant selection history are task dependent. For example, our task had multiple targets, whereas Anderson et al.'s task contained only a single, salient target; our experiment was data-limited, whereas Anderson et al.'s was response-terminated; and our effects were on target accuracy, whereas Anderson et al. failed to find any effect of irrelevant selection history on RTs.

Influence of task relevance on the costs of irrelevant selection history As in Experiment 1, the main effect of task relevance was significant $\left[F(2,32)=7.63, p=.002, \eta^{2}=.32\right]$. However, unlike in Experiment 1, the costs were numerically larger for the distractor than for the other-target condition, and then, as in Experiment 1, smallest for the flanker conditions (Fig. 5). As in Experiment 1, post-hoc pair-wise comparisons of the factors contributing to the main effect of task relevance indicated that the target performance costs in the other-target and flanker conditions were significantly different from each other, such that costs in the other-target condition were significantly larger than those in the flanker condition $\left(M_{\text {diff }}=2.9 \%\right.$, $p=.005)$, but not significantly different from those in the distractor condition $\left(M_{\text {diff }}=1.2 \%, p=.304\right)$. However, unlike in Experiment 1, the costs in the distractor condition were significantly greater than those in the flanker condition $\left(M_{\text {diff }}\right.$ $=4.1 \%, p=.004)$.

Cross-experiment comparisons There were several key differences between the pattern of costs in Experiment 3 and that observed in Experiment 1, suggesting that the manner in which task relevance and selection history interact is different when a reward history is present (Exp. 1) or absent (Exp. 3). Specifically, in Experiment 1 the pattern of costs matched our hypothesis that costs would be greatest in the other-target, smaller in the distractor, and smallest in the flanker conditions. Rather, in Experiment 3 the distractor condition was what showed the greatest costs, contrary to our hypothesis. In order (a)

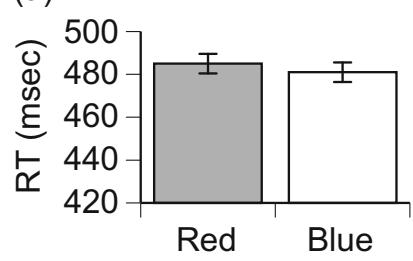

(c)

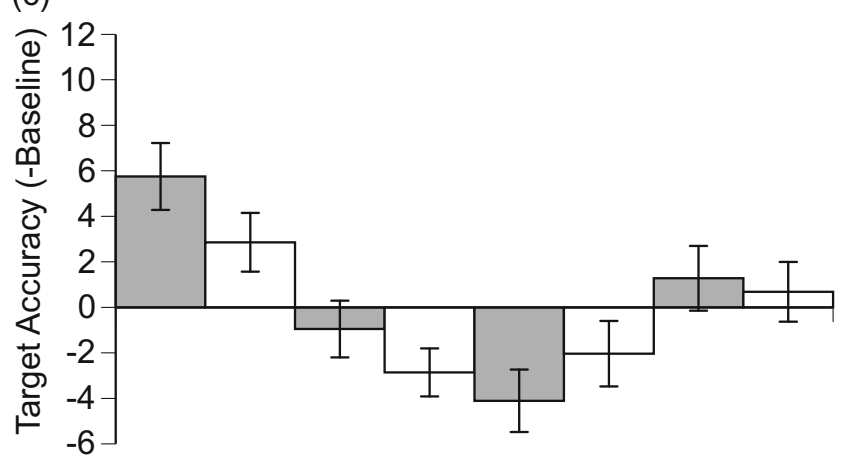

$\begin{array}{ccc}\begin{array}{c}\text { Probed } \\ \text { Target }\end{array} & \text { Other } & \text { Target }\end{array}$

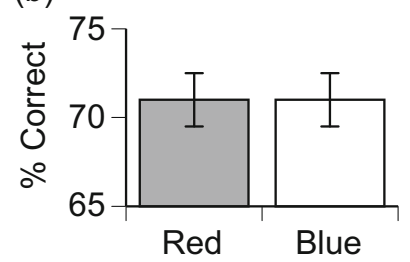

(b)

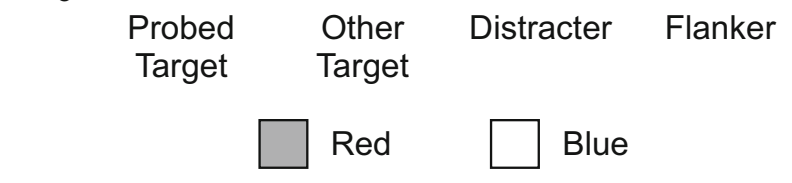

Fig. 5 Results of Experiment 3: (a) reaction time (RT) results from the training task, (b) accuracy results from the training task, and (c) baseline subtracted target accuracy results according to task relevance (probed target, other target, distractor, or flanker) and the former target feature (red or blue). Error bars represent standard errors of the mean differences (a and $\mathbf{b})$ and standard errors of the means (c) 
to determine whether the patterns of costs varied significantly as a function of experiment (i.e., Exp. 1, in which former target features were also reward-associated, vs. a control experiment, in which former target features were not rewardassociated), we performed a mixed-model ANOVA with irrelevant feature type and task relevance as within-subjects factors and Experiment (Exp. 1 vs. Exp. 3) as a between-subjects factor. For the sake of comparison, in Experiment 3 we treated the red-former-target condition as the high-reward-associated feature for half of participants, and the blue-former-target condition as the high-reward-associated feature for the other half. Note that the results were identical when the pattern was reversed and when red or blue was treated as the high-rewardassociated feature for all participants.

We observed a significant main effect of task relevance $\left[F(2,88)=9.43, p<.001, \eta^{2}=.18\right]$, although this was superseded by a significant interaction of task relevance and experiment $\left[F(2,88)=3.12, p=.049, \eta^{2}=.07\right]$. This indicates that the pattern of costs varied significantly as a function of experiment. There was also a significant interaction between feature (i.e., high/red vs. low/blue) and experiment $[F(1,44)=$ 8.03, $\left.p=.007, \eta^{2}=.15\right]$. This interaction was such that a significant difference emerged between the high- and low-reward-associated conditions in Experiment 1 (as seen previously), but no significant difference between the blue and red former target features in Experiment 3. No other main effects or interactions were significant (all $p \mathrm{~s}>.18$ ).

\section{General discussion}

The primary goal of this study was to examine whether taskirrelevant reward-associated features influence visual selection independently of task-relevant features, or whether task relevance modulates the effect of irrelevant reward-associated features. The results of both Experiment 1 and 2 supported the later hypothesis, although only when the feature was presented in task-relevant space. There were effects on performance relative to baseline when a high- but when not a lowmagnitude reward-associated feature appeared in a taskrelevant location; however, no effect was observed when the feature was presented outside of task-relevant space (flanker). Within task-relevant space, the costs of both high- and lowreward-associated features (difference from baseline) were larger when coupled with targets than with distractors that differed according to task-relevant features, although not significantly $\left(M_{\mathrm{diff}}=1.3 \%, p=.076\right.$, when collapsed across Exps. 1 and 2). Thus, the task relevance of space reliably modulates the effect of irrelevant reward-associated features, whereas the task relevance of the object itself within taskrelevant space exerts a small but unreliable influence in this task. Because the influences of task relevance within task- relevant space were equivalent for high- and low-rewardassociated features, it is possible that the effect of the irrelevant selection history of such features, and not simply of reward history, is modulated by task relevance. Consistent with this possibility, the results of Experiment 3 demonstrated that task relevance also influenced the costs observed when previously selected features were not reward-associated. However, the pattern of the effects of task relevance in Experiment 3 did not match our hypothesis, whereas those observed in both Experiments 1 and 2 did fit our hypothesis. This difference in the patterns of costs between Experiments 1 and 3 was reliable, and since the only difference between these two experiments was the presence of a reward history in Experiment 1 , it is likely that reward history accounts for this difference in the patterns of costs according to task relevance.

In order to investigate the effects of irrelevant reward associations in the context of task relevance, the design of the present study allowed the irrelevant reward-associated feature to surround different stimuli that were defined by task-relevant features, both in terms of category and space, with equal probability. The unique use of objects outside of task-relevant space (flankers), allowed us to examine the effects of irrelevant reward-associated features on attention, both within and outside of task-relevant space. Task-irrelevant reward associations do not appear to drive early visual selection outside of task-relevant space. We did not observe, in either Experiment 1 or 2, a significant effect of irrelevant learned reward in the flanker conditions, even when the flanker appeared in a previously rewarded location. This result is similar to the finding that physically salient flankers do not affect performance when they appear at a task-irrelevant location (e.g., Yantis \& Johnson, 1990), and that under conditions of high perceptual/ cognitive load, as in the present experiment, nonsalient flankers also do not affect performance (for reviews see Lavie, 2005; Lavie, Beck, \& Konstantinou, 2014). Our results indicate that irrelevant reward-associated features have reliable and robust effects inside task-relevant space. However, like physically salient features, reward-associated features are not able to overcome spatial selectivity, at least under conditions of high load. Thus, when the probability of a target in the flanker location is zero, irrelevant reward associations have no reliable effect on performance at that location in this task. Any increase in target probability at that location may influence whether or not an effect occurs, although there is evidence that the probability of a target appearing in a specific location does not modulate the effect of reward associations at that location (Stankevich \& Geng, 2014).

The results of both Experiments 1 and 2, in addition to supporting the dependent hypothesis, replicate and extend the finding that irrelevant reward-associated features impact visual selection (e.g., Anderson et al., 2011). Both experiments replicated two effects previously observed: Irrelevant reward-associated features can result in (1) costs to 
performance when they draw attention away from the target (Anderson et al., 2011; Anderson \& Yantis, 2012; Theeuwes \& Belopolsky, 2012), and (2) benefits to performance when they draw attention to the target (Della Libera \& Chelazzi, 2009). Specifically, we observed a benefit to performance when the probed target was coupled with the irrelevant reward-associated feature, and costs to performance when anything other than the probed target (other target, distractor, or flanker) was coupled with the irrelevant reward-associated feature. We also replicated the finding that these effects still occur when the reward-associated feature is physically nonsalient and when performance is no longer rewarded, indicating that the effects are not due to either the physical salience of the feature or current motivations toward reward, but rather to the irrelevant reward association itself (see Anderson, 2013, for a discussion of these issues).

The use of multiple targets in the present study created a task that placed heavier demands on perceptual processing and working memory capacity than had those used in previous studies (e.g., Anderson et al., 2011). More specifically, in the present task, optimal performance required efficient selectivity, so that multiple targets could be encoded into working memory and multiple distractors would not be. The need for a higher level of efficiency in selection due to the increased task demands relative to typical single-target designs could have reduced the influence of task-irrelevant information (e.g., Giesbrecht, Sy, Bundesen, \& Kyllingsbæk, 2014; Kyllingsbæk, Sy, \& Giesbrecht, 2011; Lavie, 2005; Lavie et al., 2014), yet the irrelevant reward-associated features continued to capture attention and impact performance. Thus, the influence of irrelevant features on visual selection - specifically, of irrelevant reward-associated features in the present study - appears even under conditions of high task difficulty.

The present study also addressed the locus of the influence of irrelevant features on visual selection. Recently, using brief, masked displays, Chelazzi and colleagues (2014) found that performance was affected by the reward history of a spatial location. Unlike unmasked stimuli presented for long durations, brief masked displays limit the availability and quality of the information to only that information that can be selected during the brief display - a data-limited design, which minimizes the relative contribution of later, postperceptual stages (Ester \& Awh, 2008; Santee \& Egeth, 1982). Furthermore, the typical use of speeded responses emphasizes response selection processes (e.g., Pashler, 1994) and introduces the possibility of data contamination by strategic speed-accuracy trade-offs and motor biases that can occur when using RTs as the dependent measure (e.g., Bisley \& Goldberg, 2006; Prinzmetal, McCool, \& Parks, 2005; Santee \& Egeth, 1982). Thus, Chelazzi et al.'s findings suggest that the effects of irrelevant reward associations in this paradigm are less likely to be due to later, postperceptual response processes. Our findings were also observed in a data-limited task and using the accuracy of an unspeeded, partial-report target identification task as the dependent measure. In other words, the design of the task in the present study emphasized the influence of early selection processes on performance, since the accuracy of target identification relied strongly on the information selected during the brief presentation of the display. Irrelevant reward-associated features continued to affect unspeeded performance in this data-limited design, indicating that the locus of the effect was less likely to be at later postperceptual or response selection stages, and more likely to be due to early selection processes.

\section{Theoretical implications}

The results of the present study support theories that learned reward associations (a) are acquired relatively quickly (800 trials in the present study) and (b) persistently (over the course of 1 week in the present study), and that they involuntarily influence selective attention in a fashion that could be contrary to the current task goals (Anderson, 2013; Anderson et al., 2011; Awh et al., 2012). In other words, the results presented here support the idea that when a particular stimulus, in this case a feature (color), is learned to be predictive of reward, that stimulus is more likely to be selected, and thus is more likely to impact performance, resulting in either benefits or costs to task performance. However, although our results do indicate that the influence of reward-associated features on attention is involuntary, since they captured attention despite instructions to ignore them, our results also indicate that the influence of the reward-associated feature is modulated by the current task relevance, most reliably by the task relevance of spatial locations.

In combination with other results showing that taskrelevant and physically salient factors influence visual selection interdependently (Nordfang et al., 2013), the results of the present study support a model of visual selective attention in which, additionally, task-relevant and -irrelevant reward-associated factors interact to weight visual information for selection. This is in line with suggestions that comprehensive models of visual selection should include a range of factors, including currently task-irrelevant ones, in order to capture the various factors that can influence visual selection (Anderson, 2013; Awh et al., 2012). This type of model complements many models of visual selective attention that accommodate the interaction of various factors (e.g., Bundesen, 1990; Desimone \& Duncan, 1995; Itti \& Koch, 2001; Kastner \& Ungerleider, 2000; Müller, Reimann, \& Krummenacher, 2003; Nordfang et al., 2013). For example, the TVA (Bundesen, 1990), with the updated formula provided by Nordfang et al., is a computational model that includes the additive and multiplicative effects of both physical salience (contrast) and relevance. The results of the present study suggest that a more comprehensive computational model would 
also include the additive and multiplicative effects of physically nonsalient irrelevant features, such as reward associations.

\section{Conclusion}

The results of the present study support the conclusion that physically nonsalient and task-irrelevant features, in this case reward-associated features, influence visual selection. We were able to observe these effects even in a task that demanded efficient selection due to the presence of multiple targets. Furthermore, the data-limited design and use of unspeeded responses have allowed us to make a stronger case that task-relevant and -irrelevant features that are physically nonsalient nonetheless influence early visual selection. Finally, the unique use of a flanker item, presented outside of task-relevant space, indicated that irrelevant features only appear to drive visual selection within task-relevant space, at least under conditions of high task difficulty. This fits with aspects of some models of visual selective attention that have suggested that task-relevant factors act to initially constrain the space in which visual selection occurs (e.g., Theeuwes, 1993, 2010; Theeuwes \& Van der Burg, 2011). Specifically, our finding that task-irrelevant reward associations do not appear to affect visual selection when they are presented outside of task-relevant space supports the idea that task relevance first limits the spatial limits of visual selection, and that visual selection proceeds within those limits. The model suggested by the present study, however, diverges from earlier models, to suggest that within task-relevant space, both relevant and irrelevant factors act interdependently to drive visual selection.

Author note This work was supported by a Postdoctoral Fellowship from the Natural Sciences and Engineering Research Council of Canada to the first author, and by the Institute for Collaborative Biotechnologies through Grant Number W911NF-09-0001 from the U.S. Army Research Office. The content of the information does not necessarily reflect the position or the policy of the U.S. Government, and no official endorsement should be inferred. We also thank Andrew Yukawa, Christine Koscheka, and Jane Lee for their help with data collection.

\section{References}

Anderson, B. A. (2013). A value-driven mechanism of attentional selection. Journal of Vision, 13(3), 7. doi:10.1167/13.3.7

Anderson, B. A., \& Folk, C. L. (2012). Dissociating location-specific inhibition and attention shifts: Evidence against the disengagement account of contingent capture. Attention, Perception, \& Psychophysics, 74, 1183-1198. doi:10.3758/s13414-012-0325-9

Anderson, B. A., Laurent, P. A., \& Yantis, S. (2011). Value-driven attentional capture. Proceedings of the National Academy of Sciences, 108, 10367-10371. doi:10.1073/pnas. 1104047108

Anderson, B. A., \& Yantis, S. (2012). Value-driven attentional and oculomotor capture during goal-directed, unconstrained viewing.
Attention, Perception, \& Psychophysics, 74, 1644-1653. doi:10. 3758/s13414-012-0348-2

Awh, E., Belopolsky, A. V., \& Theeuwes, J. (2012). Top-down versus bottom-up attentional control: A failed theoretical dichotomy. Trends in Cognitive Sciences, 16, 437-443. doi:10.1016/j.tics. 2012.06.010

Bisley, J. W., \& Goldberg, M. E. (2006). Neural correlates of attention and distractibility in the lateral intraparietal area. Journal of Neurophysiology, 95, 1696-1717. doi:10.1152/jn.00848.2005

Bundesen, C. (1990). A theory of visual attention. Psychological Review, 97, 523-547. doi:10.1037/0033-295X.97.4.523

Chelazzi, L., Eštočinová, J., Calletti, R., Gerfo, E. L., Sani, I., Della Libera, C., \& Santandrea, E. (2014). Altering spatial priority maps via reward-based learning. Journal of Neuroscience, 34, 8594 8604. doi:10.1523/JNEUROSCI. 0277-14.2014

Corbetta, M., \& Shulman, G. L. (2002). Control of goal-directed and stimulus-driven attention in the brain. Nature Reviews Neuroscience, 3, 201-215. doi:10.1038/nrn755

Della Libera, C., \& Chelazzi, L. (2009). Learning to attend and to ignore is a matter of gains and losses. Psychological Science, 20, 778-784. doi:10.1111/j.1467-9280.2009.02360.x

Desimone, R., \& Duncan, J. (1995). Neural mechanisms of selective visual attention. Annual Review of Neuroscience, 18, 193-222. doi:10.1146/annurev.ne.18.030195.001205

Ester, E. F., \& Awh, E. (2008). The processing locus of interference from salient singleton distractors. Visual Cognition, 16, 166-181. doi:10. 1080/13506280701489510

Folk, C. L., \& Remington, R. W. (2008). Bottom-up priming of top-down attentional control settings. Visual Cognition, 16, 215-231. doi:10. 1080/13506280701458804

Giesbrecht, B., Sy, J., Bundesen, C., \& Kyllingsbæk, S. (2014). A new perspective on the perceptual selectivity of attention under load. Annals of the New York Academy of Sciences, 1316, 71-86. doi: 10.1111/nyas. 12404

Hickey, C., Chelazzi, L., \& Theeuwes, J. (2014). Reward-priming of location in visual search. PloS One, 9, e103372. doi:10.1371/ journal.pone. 0103372

Itti, L., \& Koch, C. (2001). Computational modelling of visual attention. Nature Reviews Neuroscience, 2, 194-203. doi:10.1038/35058500

Kastner, S. K., \& Ungerleider, L. G. (2000). Mechanisms of visual attention in the human cortex. Annual Review of Neuroscience, 23, 315 341. doi:10.1146/annurev.neuro.23.1.315

Kiss, M., Driver, J., \& Eimer, M. (2009). Reward priority of visual target singletons modulates event-related potential signatures of attentional selection. Psychological Science, 20, 245-251. doi:10.1111/j.14679280.2009.02281.x

Kyllingsbæk, S., Sy, J. L., \& Giesbrecht, B. (2011). Understanding the allocation of attention when faced with varying perceptual load in partial report: A computational approach. Neuropsychologia, 49, 1487-1497. doi:10.1016/j.neuropsychologia.2010.11.039

Lavie, N. (2005). Distracted and confused? Selective attention under load. Trends in Cognitive Sciences, 9, 75-82. doi:10.1016/j.tics. 2004.12.004

Lavie, N., Beck, D. M., \& Konstantinou, N. (2014). Blinded by the load: Attention, awareness and the role of perceptual load. Philosophical Transactions of the Royal Society B, 369, 20130205. doi:10.1098/ rstb.2013.0205

Monsell, S. (2003). Task switching. Trends in Cognitive Sciences, 7, 134140. doi:10.1016/S1364-6613(03)00028-7

Müller, H. J., Reimann, B., \& Krummenacher, J. (2003). Visual search for singleton feature targets across dimensions: Stimulus- and expectancy-driven effects in dimensional weighting. Journal of Experimental Psychology: Human Perception and Performance, 29, 1021-1035. doi:10.1037/ 0096-1523.29.5.1021 
Nordfang, M., Dyrholm, M., \& Bundesen, C. (2013). Identifying bottomup and top-down components of attentional weight by experimental analysis and computational modeling. Journal of Experimental Psychology: General, 142, 510-535. doi:10.1037/a0029631

Pashler, H. (1994). Divided attention: Storing and classifying briefly presented objects. Psychonomic Bulletin \& Review, 1, 115-118. doi:10. 3758/BF03200766

Prinzmetal, W., McCool, C., \& Park, S. (2005). Attention: Reaction time and accuracy reveal different mechanisms. Journal of Experimental Psychology: General, 134, 73-92. doi:10.1037/ 0096-3445.134.1.73

Raymond, J. E., \& O’Brien, J. L. (2009). Selective visual attention and motivation the consequences of value learning in an attentional blink task. Psychological Science, 20, 981-988. doi:10.1111/j.1467-9280. 2009.02391.x

Santee, J. L., \& Egeth, H. E. (1982). Do reaction time and accuracy measure the same aspects of letter recognition? Journal of Experimental Psychology: Human Perception and Performance, 8, 489-501. doi:10.1037/0096-1523.8.4.489

Stankevich, B. A., \& Geng, J. J. (2014). Reward associations and spatial probabilities produce additive effects on attentional selection. Attention, Perception, \& Psychophysics, 76, 2315-2325. doi:10. 3758/s13414-014-0720-5
Sy, J. L., Elliott, J. C., \& Giesbrecht, B. (2013). Post-perceptual processing during the attentional blink is modulated by inter-trial task expectancies. Frontiers in Human Neuroscience, 7, 627. doi:10.3389/ fnhum.2013.00627

Theeuwes, J. (1992). Perceptual selectivity for color and form. Perception \& Psychophysics, 51, 599-606. doi:10.3758/BF03211656

Theeuwes, J. (1993). Visual selective attention: A theoretical analysis. Acta Psychologica, 83, 93-154. doi:10.1016/0001-6918(93)90042-P

Theeuwes, J. (2010). Top-down and bottom-up control of visual selection. Acta Psychologica, 135, 77-99. doi:10.1016/j.actpsy.2010.02.006

Theeuwes, J., \& Belopolsky, A. V. (2012). Reward grabs the eye: Oculomotor capture by rewarding stimuli. Vision Research, 74, 80-85. doi:10.1016/j.visres.2012.07.024

Theeuwes, J., Kramer, A. F., \& Belopolsky, A. V. (2004). Attentional set interacts with perceptual load in visual search. Psychonomic Bulletin \& Review, 11, 697-702. doi:10.3758/BF03196622

Theeuwes, J., \& Van der Burg, E. (2011). On the limits of top-down control of visual selection. Attention, Perception, \& Psychophysics, 73, 2092-2103. doi:10.3758/s13414-011-0176-9

Yantis, S., \& Johnson, D. N. (1990). Mechanisms of attentional priority. Journal of Experimental Psychology: Human Perception and Performance, 16, 812-825. doi:10.1037/0096-1523.16.4.812 